Vol. 25, n. 1, 2020.

Artigo recebido: 03/02/2020

Artigo aprovado em: 04/03/2020

\title{
DESDE LA CONSTRUCCIÓN DE PROYECTOS AMBIENTALES AL EMPODERAMIENTO Y RESCATE AMBIENTAL
}

\author{
Andrea Castillo Rodas ${ }^{1}$
}

\section{RESUMO}

Este trabalho investiga as políticas públicas que levaram à construção de projetos de educação ambiental (PRAE) na Colômbia, bem como a realização da análise do PRAE de uma instituição educacional, à luz das diversas teorias sobre o meio ambiente de autores como Lucie Sauve, Enrique Leff e Bronferbrenner. Nesse sentido, a história da EA (educação ambiental) permite esclarecer as etapas que levaram à construção desta pedagogia, em uma análise das leis colombianas, pode-se concluir que as ferramentas necessárias foram fornecidas desde a política construção dos projetos, no entanto, quando são analisados, há varias lacunas em termos de corpos teóricos e relações integrais que tem em seu desenvolvimento e nas atividades propostas com a integralidade do ser ou formação do cidadão ambiental , limitada à gestão dos recursos naturais, por fim, esta análise permite propor um projeto ambiental escolar a partir da integralidade do ser e do seu ambiente (o eu, o outro e o meio ambiente).

Palavras-chave: projeto ambiental escolar (PRAE), educação ambiental, diálogo do conhecimento e racionalidade ambiental

\footnotetext{
${ }^{1}$ Docente de Ciencias Naturales, Licenciada en Biología Universidad Distrital Francisco José de Caldas, Master em didáctica de las Matemáticas em Educación Infantil y Primaria, Universidad Internacional de la Rioja, Estudiante de Maestría em Docencia de la Química, Universidad Pedagógica Nacional de Colombia. Correo electrónico: acastillor@upn.edu.co
} 
AMBIENTE \& EDUCAÇÃO

E-ISSN - 2238-5533

v. 25, n.1, 2020

\section{FROM THE CONSTRUCTION OF ENVIRONMENTAL PROJECTS TO EMPOWERMENT AND ENVIRONMENTAL RESCUE}

\section{ABSTRACT}

This brief investigates the public policies that have led to the construction of environmental education projects (PRAE) in Colombia, as well as conducting the analysis of an education al institution's PRAE, in the light of the various theories on the environment of autor ssuch as Lucie Sauve, Enrique Leff and Bronferbrenner. In this sense the history of the EA (Environmental Education) allow stoclarify the step sthat led to the construction of this pedagogy, in an analysis of Colombian law sit can be concluded that the necessary tolos have been provided since the political construction of the projects, however, whenthey are analyzed there are several gaps in terms of theoretical bodies and integral relation shipthatit has in its development and in the proposed activities with the integrality of being or training of the environmental citizen, limited to the management of natural resources. Finally this analysis allows to propose a School Environmental Project from the integrality of being and its environment (the self, the other and the environment).

Keywords: School Environmental Project (PRAE), Environmental Education, Knowledge Dialogue and Environmental Rationality

\section{INTRODUCCIÓN}

El presente trabajo es un análisis de la política pública que permitieron el nacimiento existencia de los PRAES (Proyectos Educativos Ambientales) en las instituciones educativas de Colombia, en un principio se muestran los referentes teóricos que permiten entender el porqué de una educación ambiental a nivel global y nacional. A partir del concepto integrador de Lucie Sauve de educación ambiental se hace un análisis de las políticas públicas nacionales que son la guía para la realización de los Proyectos ambiéntales en las instituciones educativas del país.

En este primer análisis se concluye que existen un marco legal muy amplio que permite llevar a cabo proyectos educativos ambientales, pero son pocas las garantías en términos de apoyos académicos o económicos que reciben las instituciones para la ejecución de dichos proyectos. 
En un segundo apartado se analizan 3 experiencias exitosas en la implementación a nivel nacional de los proyectos educativos ambientales buscando puntos en común entre estos para posteriormente plantear un PRAE que tenga verdadero impacto social y ambiental desde las instituciones, en este sentido los puntos en común entre los tres proyectos fueron:

1. Las problemáticas tenidas en cuenta y las cuales se pretenden solucionar surgen de las necesidades de la comunidad y no desde intereses o visiones particulares de agentes externos,

2. Los proyectos generados permiten las alianzas con entes gubernamentales e instituciones educativas como universidades,

3. Se tienen en cuenta los factores históricos y sociales que han conducido a la problemática así como los factores que afectan la parte socio- cultural de las comunidades,

4. Se plantean estrategias en la formación de valores que permitan un cambio de actitud frente al ambiente y el trabajo en equipo,

5. Se sistematizan y evalúan con la comunidad la pertinencia de los proyectos y de esta manera se actualizan entre toda la comunidad educativa para que sigan generando el impacto esperado, estos puntos se amplían más adelante.

Finalmente se hace un tercer análisis sobre el PRAE de la institución educativa distrital Colegio Villas del Progreso, y se realiza una propuesta acorde con las necesidades de la comunidad que brinde un verdadero empoderamiento, apropiación y rescate ambiental no solo de los estudiantes sino de toda la comunidad circundante con el colegio.

\section{REFERENTES TEÓRICOS}

A pesar de la preocupación común que existe en la Educación Ambiental EA por superar las dificultades y crisis ambientales y ecológicas que han surgido en los últimos tiempos, los diversos actores (investigadores, profesores, pedagogos, animadores, asociaciones, organismos, etc.) presentas discursos diferentes y concepciones distintas sobre la educación ambiental que están 
permeadas por la visión o concepto de ambiente que maneja cada uno; (Sauve, 2005) es por esto que a continuación se presenta una cartografía del concepto de ambiente planteada por Lucie Sauve que servirá de base para entender el análisis de los PRAE y la formulación de la nueva propuesta.

\section{El Concepto de Ambiente}

Antes de hablar de educación ambiental es importante conocer las diferentes posturas sobre el concepto de ambiente en este sentido Sauve (2005) plantea una tipología de la concepción de ambiente en la educación ambiental, sobre la cual analizaremos posteriormente un conjunto de proyectos ambientales (cuadro 1)

\begin{tabular}{|c|c|c|c|}
\hline Ambiente & Tipos de Relación & Características principales & $\begin{array}{l}\text { Ejemplos de estrategias de } \\
\text { enseñanza /aprendizaje }\end{array}$ \\
\hline Como naturaleza & $\begin{array}{l}\text { Para ser respetada } \\
\text { preservada y } \\
\text { conservada }\end{array}$ & $\begin{array}{l}\text { La naturaleza como el centro, el } \\
\text { origen, la matriz, el útero que nos } \\
\text { acoge a todos. }\end{array}$ & $\begin{array}{l}\text { Estar inmerso en la naturaleza } \\
\text { Admirar la naturaleza }\end{array}$ \\
\hline Como un recurso & Para ser Manejado & $\begin{array}{l}\text { Nuestra herencia colectiva, calidad } \\
\text { sustentable de vida }\end{array}$ & $\begin{array}{l}\text { Campaña de las } 3 R \text { s } \\
\text { Control de manejo de los } \\
\text { recursos de energía y agua }\end{array}$ \\
\hline Como un problema & Para ser resuelto & $\begin{array}{l}\text { El ambiente biofísico, el soporte, la } \\
\text { amenaza por la contaminación y el } \\
\text { deterioro }\end{array}$ & $\begin{array}{l}\text { Estrategia de solución de } \\
\text { problemas; estudios de caso }\end{array}$ \\
\hline $\begin{array}{l}\text { Como un lugar } \\
\text { donde vivir }\end{array}$ & $\begin{array}{l}\text { Para conocerlo, } \\
\text { aprender de él y } \\
\text { conocerlo }\end{array}$ & $\begin{array}{l}\text { Nuestro ambiente diario con su socio } \\
\text { - cultura, sus componentes } \\
\text { tecnológicos e históricos }\end{array}$ & $\begin{array}{l}\text { Historia Ambiental de nuestra } \\
\text { comunidad; proyectos de eco- } \\
\text { arbolado. }\end{array}$ \\
\hline Como la Biosfera & $\begin{array}{l}\text { En el cual todos } \\
\text { vivimos juntos en el } \\
\text { futuro }\end{array}$ & $\begin{array}{l}\text { La tierra como nave espacial, objeto } \\
\text { de la conciencia planetaria, un mundo } \\
\text { de interdependencias entre los seres } \\
\text { vivos y la cosas }\end{array}$ & $\begin{array}{l}\text { Estudios de caso sobre temas } \\
\text { globales; foros ambientales. }\end{array}$ \\
\hline $\begin{array}{l}\text { Como un proyecto } \\
\text { de la comunidad }\end{array}$ & $\begin{array}{l}\text { En el cual se está } \\
\text { involucrado }\end{array}$ & $\begin{array}{l}\text { Un entorno vivo compartido, el foco } \\
\text { del análisis sociocrítico; una } \\
\text { preocupación política para la } \\
\text { comunidad }\end{array}$ & $\begin{array}{l}\text { Investigación integral en acción } \\
\text { (procesos de participación } \\
\text { dirigido a la transformación); } \\
\text { narraciones de cuentos para } \\
\text { ilustrar diferentes } \\
\text { cosmovisiones }\end{array}$ \\
\hline
\end{tabular}

Cuadro 1. Tipología de la concepción de ambiente según Lucie Sauve (2005)

Desde esta perspectiva del concepto de ambiente dentro de la educación ambiental es posible analizar las prácticas ambientales que lleven actualmente en la escuela y que generan visiones y relaciones con el entorno, para el fin de este análisis de PRAES se quiere llegar a una visión integradora del ambiente dentro de la educación ambiental que a su vez sea transformadora de las ideas y de las 
practicas que se dan en las instituciones educativas, viendo el ambiente como proyecto de comunidad, biosfera y lugar donde vivir, mediante estrategias como las plantadas por Sauve en su cartografía y otras a fines con esas ideas de ambiente.

De acuerdo con las diferentes corrientes antes expuestas los actores involucrados en el proceso de la EA conciben el ambiente y generan prácticas en relación con dicha corriente (Sauve, 2007).

\section{¿POR QUÉ DE LA EDUCACIÓN AMBIENTAL?}

En principio las sociedades humanas se encontraban en contacto directo con la naturaleza y coexistían en armonía con ella, sin embargo, la paulatina urbanización de las poblaciones humanas y su asentamiento llevo al hombre a alejarse de la naturaleza, convirtiéndose en un sujeto antropocéntrico, cuyo interés era el de satisfacer sus necesidades, sin tener en cuenta el mundo que le rodea siguiendo los ideales del desarrollo económico. Dentro de esta lógica del desarrollo económico ha visto a la naturaleza como el proveedor de recursos los cuales pueden ser explotados según las necesidades y disposiciones de los humanos, llevando al planeta a una crisis ecológica y ambiental que afecta a todas las formas de vida existentes (García y Ferrandis, 1990).

De acuerdo con lo anterior la educación ambiental surge de la necesidad de sensibilizar y actuar frente a la crisis ambiental que sufre el planeta, esta sensibilización busca generar relaciones armónicas de los individuos consigo mismo, con los otros y con su entorno; de manera local y global. Así mismo, las EA (Educación Ambiental) busca resignificar las relaciones entre los hombres y el ambiente, generando interacciones más acordes con las dinámicas ambientales. En palabras de Novo (2006) el cambio en la percepción general de las relaciones hombre - naturaleza provoca, lógicamente, una nueva visión pedagógica del tema ambiente.

Desde este cambio de perspectiva lo primero será volver a solucionar los problemas de manera integral, unificar las ciencias sociales y las ciencias 
ambientales, pues como ya se ha visto los problemas ambientales son producto de la intervención del hombre y el afloramiento de las culturas, sin embargo; no podemos ser extremistas y quitarle al hombre su cultura en pro de la conservación ambiental, es necesario evaluar todos los factores que nos llevaron como civilización a hasta este punto de crisis ambiental, es así que la educación ambiental debe estar encaminada a integrar la historia, la ciencias naturales, la economía, la cultura y hasta la psicología para entender los comportamientos y emociones respecto al ambiente que presentan los seres humanos.

De acuerdo con este último aspecto es importante integrar la dimensión personal, es decir, lo psicológico, lo espiritual y emocional de cada ser humano, puesto que la visión y la forma, en la cual este se relaciona, consigo mismo y con otros (entiéndase el otro como ser vivo y ambiente), determinará la forma en la cual establece estructuras mentales respecto al ambiente, como da valor, sentido y significado a aquello con lo que se relaciona.

Así podemos definir la educación ambiental como una práctica de formación social y ciudadana de tipo transdisciplinar, que permite a los individuos una relación universal con el ambiente, promoviendo el diálogo de saberes, la protección y conservación de los recursos y el respeto por el ambiente, entendiendo el ambiente en términos de las relaciones consigo mismo, los otros, y sus diversos entornos, como lo muestra Sauve (2005).

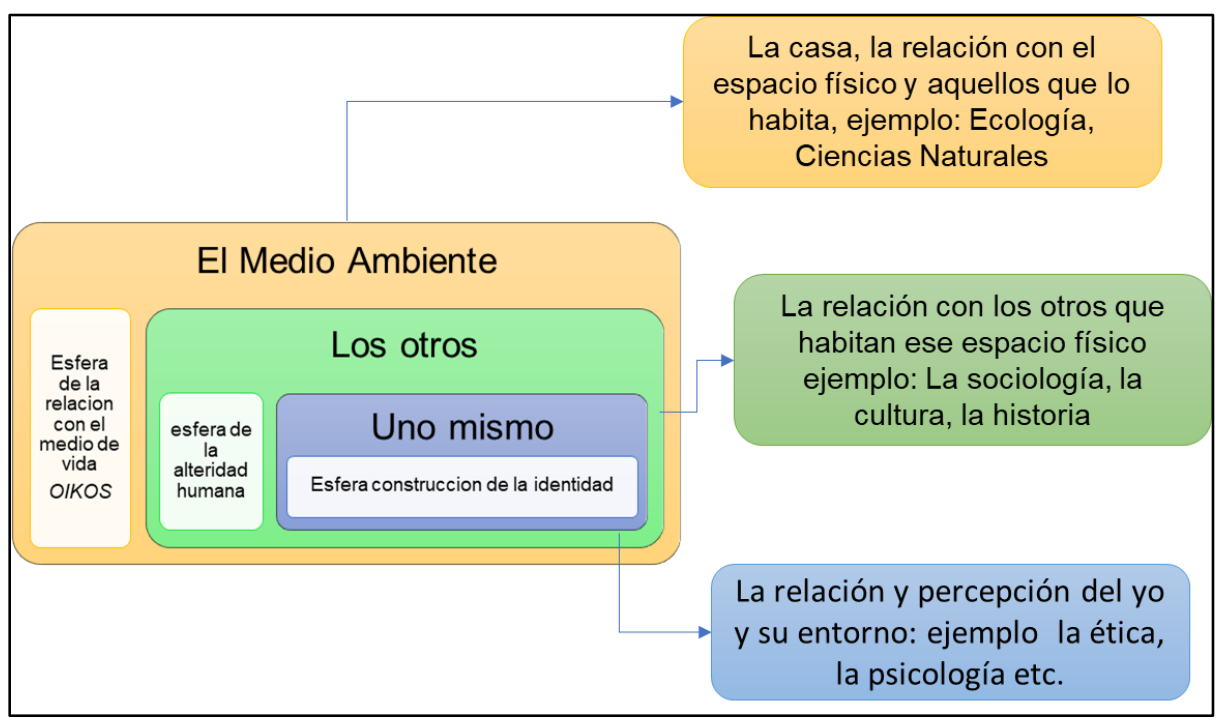


Figura 1 El concepto de Ambiente Tomado y adaptado de Sauve (2005)

Es así como la educación ambiental no solo se preocupa por los procesos de recuperación de los espacios físicos sino también de los territorios como espacios de formación de la cultura, podemos decir que la educación ambiental tiene la misión de fortalecer los lazos entre los territorios y saberes ancestral, físico, social y emocional, llegando a consensos que permitan la solución de todos los problemas ambientales presentes en dichos espacios, desde las diferentes disciplinas que aportan a la construcción de los territorios.

\section{COLOMBIA Y LA EDUCACIÓN AMBIENTAL}

En Colombia se empieza a hablar sobre educación ambiental hacia la década de los 70, con el Código Nacional de los Recursos Naturales Renovables y la Protección al Medio Ambiente en diciembre de 1974 (MINAMBIENTE "Ministerio de Ambiente y Desarrollo Sostenible de Colombia"), este presenta al ambiente como patrimonio común en donde las personas y el estado deben velar por la protección y conservación de los ecosistemas, con este fin el estado hizo uso de los medios de comunicación nacional para informar y concientizar sobre las problemáticas ambientales de tipo local, nacional y regional (MINAMBIENTE, 1974).

En el código también se busca integrar la EA dentro de la educación formal, con el fin de generar estrategias y espacios destinados a el reconocimiento de las situaciones ambientales, apropiación del territorio, entender la relación del hombre con el ambiente y construir espacios que permitan entender de manera científica el funcionamiento de los ecosistemas colombianos para su protección y conservación (MINAMBIENTE, 1974).

La ley 99 de 1993, recoge los acuerdos de Rio de Janeiro de 1992, esta ley contempla el ambiente y el desarrollo, mediante la formulación de proyectos educativos y ambientales enfocados hacia la Cultura de la paz, estos proyectos debían ser propuestos desde el reconocimiento de las problemáticas ambientales 
y la participación de la ciudadanía. De esta ley se nace en octubre el decreto 1743 de 1994, el cual reglamenta la existencia de los PRAE (Proyectos Escolar de Educación Ambiental), este tiene la finalidad de formar ciudadanos críticos y reflexivos sobre el ambiente y la practicas que se desarrollan en él, así mismo los PRAE buscan el reconocimiento de las problemáticas ambientales locales, que permitan generar propuestas que permitan solucionar 0 mitigar dichas problemáticas desde el ejercicio académico y las relaciones de la comunidad con la escuela y el ambiente. De acuerdo con esto el ministerio de ambiente y desarrollo sostenible, y la subdirección de educación y participación han ajustado las metodologías y formulación de los PRAE en el país (MINAMBIENTE, 1994).

En el 2002 se propone para Colombia la Política de Educación Ambiental SINA, la cual rige las intervenciones sobre el ambiente y da las directrices sobre EA. Este documento se enfoca en las características del contexto colombiano y genera cuatro objetivos que le permitirán al país avanzar a nivel de Ambiente estos son:

- Propender por la actualización continua de conceptos en materia de medio ambiente dentro de todo el sector educativo.

- Incluir de manera transversal la educación ambiental en todos los sectores.

- Establecer instrumentos de diálogo con la comunidad a fin de crear modelos de desarrollo que contribuyan con la sostenibilidad.

- Fomentar en cada proceso la búsqueda del equilibrio entre la sociedad, la cultura y el ambiente a fin de mantener el concepto de sostenibilidad.

Para el alcance de estos objetivos se propone fortalecer las entidades y proyectos que participan del proceso en todos los niveles: Comités Técnicos Interinstitucionales de Educación Ambiental (CIDEA), ejecución de Proyectos ciudadanos de Educación Ambiental (PROCEDAS), y el planteamiento y desarrollo de los proyectos ambientales escolares (PRAES, MINAMBIENTE, 2002).

A pesar del planteamiento de la ley ambiental es indiscutible que en Colombia no existe una cultura ambiental generalizada por lo cual es necesario seguir fortaleciendo los proyectos y entidades que participan de estos procesos, 
esto requiere de un gran esfuerzo por parte de los educadores ambientales en cuanto a la formación de ciudadanos ambientales (MINAMBIENTE, 2002).

Desde la política pública Colombiana se ve el ambiente como recurso, como naturaleza y como problema, lo que guía los PRAES a la gestión de los recursos, la resolución de problemas de contaminación y la apreciación por la naturaleza, si bien estos puntos son importantes para la construcción de las sociedades, no son los únicos factores que se deben tratar en la EA, por los cual las políticas públicas también deben apuntar a la integralidad del ser y su relación como se dijo antes del yo, el otro y su entorno. A pesar de esto los PRAES brindan herramientas valiosas que pueden ser útiles en la construcción de nuevas formas de ver la educación ambiental en el país teniendo claro el respeto por la otredad y las diversas formas de ver esa relación hombre - naturaleza (Sauve, 2005; Leff, 2012).

Al ver el ambiente como naturaleza, recurso y problema, las políticas públicas se quedan cortas y desconocen los saberes presentes en las diferentes culturas, olvidan el ambiente como otro con el cual convivir y se generan presiones entre las comunidades que habitan las zonas y los encargados de la conservación y protección del espacio físico al no entender las dinámicas socio - culturales que atraviesan a dicho territorio (Leff, 2012).

\section{¿EL PRAE UNA SOLUCIÓN A LA CRISIS AMBIENTAL?}

Los de PRAE (Proyecto Escolar de Educación Ambiental), se presenta como respuesta a las leyes 115 de 1994 (Ley General de Educación) y la 99 de 1993 (Ley General Ambiental de Colombia) y nacen en Colombia el 5 de agosto de 1994 con el decreto 1743 de 1994, como parte de la formación integral de los ciudadanos, los ministerios de Ambiente y de Educación Nacional, se integran en este para generar alternativas de formación hacia la construcción de relaciones armónicas con el ambiente, crear conciencia sobre la protección, conservación del ambiente, mejoramiento de la calidad de vida y el uso racional de los recursos naturales. Con lo anterior, el decreto pide instaurar de manera obligatoria los 
PRAE en todas las instituciones del país, al igual que en todos sus niveles de formación preescolar, básica y media.

Estos proyectos educativos ambientales serán una construcción colectiva (diseño y desarrollo) que involucrara a toda la comunidad (padres, estudiantes, directivos y sectores productivos), sus objetivos principales serán:

1. Realizar un diagnóstico ambiental de la zona donde se llevara a cabo el proyecto,

2. Reconocer los problemas ambientales que afectan a la comunidad y

3. la resolución de dichos problemas. Teniendo en cuenta la cultura, la sociedad, la naturaleza y las tradiciones propias del quienes allí habitan, teniendo en cuenta los principios de interculturalidad, formación de valores, regionalización (apropiación y pertenencia al territorio), interdisciplinaridad, participación democrática, formación política y gestión.

Sera la comunidad la encargada de liderar estos proyectos con apoyo de los educadores ambientales, el gobierno escolar prestara su apoyo para la difusión y generación de los canales de participación durante todo el proceso, y las entidades ambientales y secretarias de educación de cada región brindaran su apoyo en capacitación y actualización, los ministerio de ambiente y desarrollo sostenible y el ministerio de educación serán los encargados de dar las directrices que permitan la ejecución de los proyectos, finalmente el IDEAM (Instituto de Hidrología, Meteorología y Estudios Ambientales) y las instituciones de educación informaran sobre las nuevas tecnología y conocimiento en materia de ambiente a las secretarias de educación para que estos sean tenidos en cuenta por las instituciones educativas en la formación y continuación de los proyectos.

En cuanto a la evaluación los PRAE, deben evaluar:

1. el impacto social, ambiental y cultural,

2. Como ha mejorado la calidad de vida,

3. Y como se ha dado solución a los problemas locales. Esta evaluación debe ser periódica y realizarse mínimo 1 vez al año.

Aunque la ley es clara en los aspectos y responsabilidades que deben ser asumidas por todos los actores dentro de los PRAE, son pocos los proyectos que 
logran un impacto ambiental real, es por lo que se analizaran algunas experiencias significativas de PRAE, divulgadas por el MEN (Ministerio de Educación Nacional) para ver sus fortalezas y debilidades y a partir de este marco de referencia se analizara el PRAE de la I.E.D. Colegio Villas del Progreso de la ciudad de Bogotá.

1. PRAE, Arroyando: escuela y comunidad caminando hacia la sostenibilidad del agua y el suelo de su municipio (Institución Educativa Pijiguayal)

2. PRAE, Vivir en comunidad un compromiso de todos hacia la sostenibilidad ambiental de Sandoná (Institución Educativa Sagrado Corazón de Jesús)

3. PRAE, Mientras Cambia la Escuela: el Conocimiento del territorio se Incorpora Efectivamente en la Formación de una ciudadanía Ambiental (Normal Superior de Popayán)

Después, de la lectura de los tres proyectos ambientales escolares antes mencionados, son varios los aspectos en los que coinciden, a la hora de formular y desarrollar sus proyectos ambientales entre estos se destacas:

1. El reconocimiento de una problemática ambiental, que afecte de manera directa o indirecta a la institución educativa a su población circundante, muchos de los proyectos toman el agua como un recurso importante en las comunidades y a partir de este eligen una problemática, lo mismo ocurre con el suelo y el uso de los suelos en actividades económica, sociales y culturales

2. La construcción conjunta de toda la comunidad educativa, los proyectos aquí planteados tienen pertinencia en los contextos donde se desarrollan y afectan a la comunidad, por lo cual integran a estudiantes, docentes, directivos docentes, pobladores y sectores productivos

3. Las instituciones educativas han generado alianzas con entidades que les permiten una mayor comprensión del problema ambiental a solucionar, también les permiten la formación continua en términos de ambiente, procesos tecnológicos, avances científicos, delimitación leal entre otros.

4. Se mueven bajo la relación sociedad, cultura y naturaleza; entendiendo que las situaciones ambientales dependen de muchos factores por lo cual no se 
pueden tratar desde una sola rama del conocimiento, deben abarcar la interdisciplinaridad entre las diversas ciencias y formas del saber.

5. Indagan sobre los procesos históricos, políticos, económicos y culturales que han llevado al surgimiento de la problemática a estudiar.

6. Plantea estrategias de formación de ciudadanos en valores respecto al ambiente y al entendimiento de su entorno, y como se puede establecer un equilibrio ambiente - sociedad, a partir de la implementación de proyectos e investigaciones del contexto, que permitan modificar prácticas culturales y la apropiación del territorio.

7. Se presentan como proyectos que permiten el aprovechamiento adecuado del ambiente, y la conservación y protección de los recursos naturales, por lo cual no tiene una visión centrada en el respeto del ambiente si no en el aprovechamiento.

8. Cuentan con un enfoque pedagógico y didáctico, sin embargo, hace falta explicitar cuales son los enfoques social, económico y ambiental que se pretenden con la ejecución de los proyectos.

9. La sistematización continua de los proyectos ha permitido conocer las fortalezas y debilidades que este plantea es por eso por lo que se hace necesario dicho proceso dentro de la evaluación de las experiencias exitosas y su impacto en la escuela y la comunidad.

10. Aunque son proyectos muy completos y da alto impacto en la comunidad, debería existir una parte que se preocupe por la dimensión del yo, como es la relación de los estudiantes con sus estructuras, mentales, emocionales y cognitivas.

Desde esta perspectiva los proyectos analizados ven al ambiente como un lugar donde vivir y que será la futura casa de todas las formas de vida que allí conviven, también es un proyecto de comunidad ya que tiene en cuenta las necesidades de quienes allí viven y se generan diálogos de saberes entre los diferentes pobladores, lo que puede permitir en el futuro un cambio de la visión sobre el ambiente más integral y comprometida. 
Acordes con esta visión de ambiente todos los participantes de la comunidad tienen un papel activo dentro del proyecto y ven al ambiente como otro en si con el cual se han generado relaciones armónicas.

\section{ANÁLISIS Y PRESENTACIÓN DEL PRAE INSTITUCIÓN EDUCATIVA - DISTRITAL COLEGIO VILLAS DEL PROGRESO²}

Descripción de la Población

El colegio VILLAS DEL PROGRESO I.E.D. está ubicado en la ciudad de Bogotá - Colombia, cuenta con dos sedes las cuales para el 2018 se encontraban aproximadamente 4000 estudiantes matriculados desde el grado de jardín, hasta grado once, en edades que oscilan entre los 4 y los 19 años. La mayoría de los estudiantes pertenecen a los estratos $0,1,2$ y 3 muchos de hechos no son originarios de la ciudad de Bogotá, sino de otros sitios como la costa Caribe, pacifica, los llanos orientales etc. Para el presente año la población de origen venezolana aumento considerablemente, lo que convierte al colegio Villas del progreso en un escenario multiétnico y pluricultural. (Figura 2)

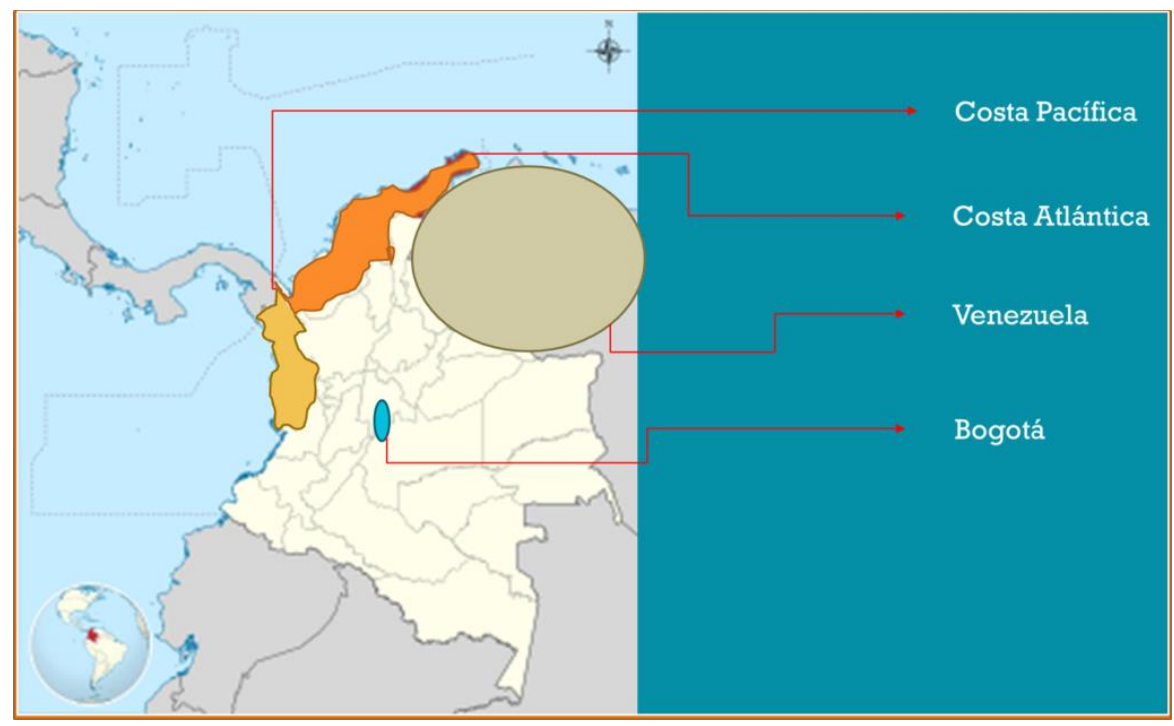

\footnotetext{
${ }^{2}$ Generando cultura frente al manejo de residuos mejoro mi entorno y protegemos nuestros recursos, es el subtitulo del proyecto.
} 
Figura 2. Procedencia de la población estudiantil del Colegio Villas del Progreso

\section{EI PRAE actual}

EI PRAE de la institución Educativa Distrital Colegio Villas del Progreso se presenta como un proyecto en caminado al uso adecuado de los recursos naturales como problemática ambiental a desarrollar, dicha problemática fue puesta en evidencia por los docentes del área de ciencias naturales y educación ambiental de la institución y es liderada por los mismos. Desde esta perspectiva se plantea la generación de subproyectos encaminados a desarrollar destrezas, habilidades y valores frente al entorno el manejo de los recursos naturales así se plantean 4 problemáticas que son:

Manejo de los Residuos Sólidos: Para su solución se plantea el uso de las tres Rs, reciclar, reducir y reutilizar y la fabricación de compost para ser aprovechado en la huerta escolar (aunque esta huerta escolar no aparece en el documento de PRAE)

Falta de Espacios Verdes: Generación de un muro verde, la cerca viva y ubicación de macetas dentro de la institución

El Usos y Aprovechamiento del Agua: Concientizar a los estudiantes sobre el uso del agua y su aprovechamiento para que no jueguen con el recurso

La Calidad del Aire: que se genere conciencia sobre como la calidad del aire afecta la calidad de la vida

Conociendo estas problemáticas y posibles soluciones los docentes propone estrategias que permitan alcanzar los objetivos, generando nuevas formas de comportamientos y actitudes frente a su entorno cercano.

en coherencia con lo expuesto en los marcos internacionales las teorías expuestas podemos decir que este PRAE ve el ambiente como un recurso, donde es necesario su buen manejo para mejorar la calidad de vida de los seres humanos y como naturaleza que debe ser apreciada, admirada y conservada, sin embargo es evidente que esta visión de ambiente se queda corta y no tiene en cuenta las 
diferentes circunstancias y cosmovisiones que han llevado al problema de la comunidad, tampoco tienen en cuenta las necesidades de toda la comunidad si no que parte de la percepción de uno pocos que no son tan cercanos si no pasajeros en este espacio tiempo.

A comparación de los PRAE antes expuestos es evidente la carecía en ciertos aspectos que son importantes en los proyectos ambientales como son

La generación de alianzas con entidades externas de tipo ambiental o de educación superior que capaciten y formen sobre las cuestiones ambientales.

Existe un desconocimiento de las causas históricas, políticas, sociales, económicas y culturales que han generado las problemáticas ambientales actuales. Así como las características ecológicas y físicas del ambiente que permitan entender de manera científica como el entorno se están viendo afectado por dichas problemáticas.

En cuestión de la comunidad no existe una construcción colectiva ya que tanto el reconocimiento de los problemas, como estrategias de solución de estos son propuestas y aplicadas por el cuerpo docente que en su mayoría no pertenece a la comunidad, por lo cual los sub proyectos planteados no generan mayor impacto en la comunidad si no que son vistos como otro tipo de actividades académicas.

No existe un diagnóstico de las problemáticas desde las necesidades de la población, es por esto por lo que los proyectos no han generado el impacto socio - ambiental que pretenden.

Se menciona la condición de desplazamiento de gran parte de la población es decir muchos de los estudiantes no pertenecen a la ciudad, por lo cual necesitan generar nuevas relaciones de pertenencia con el ambiente, y mirar como la percepción sobre el nuevo entorno le permite establecer relaciones de manera positiva con el ambiente y los otros.

A pesar de las dificultades evidentes en la construcción del proyecto es importante destacar, que es cierto grado los estudiantes han generado 
comportamientos sobre el cuidado del entorno, sin embargo, estos no han sido significativos o han trascendido a los hogares o a los sectores productivos cercanos al colegio.

Desde la perspectiva de Leff (2004), la educación ambiental tiene el papel de cambiar la racionalidad sobre el ambiente, reconocer en el ambiente la presencia de otro, la otredad desde la igualdad y respeto por su existencia, en este sentido la formulación de los PRAE aquí expuesto, está determinada por una visión de capital, en donde el ambiente no es otro si no un recurso del cual se beneficia la vida humana, si bien se piensa en generar acciones y estrategias que permitan un mejoramiento del medio ambiente, las actividades propuestas en todos los casos no cambian la racionalidad sobre el ambiente.

En cuanto al dialogo de los saberes, los primeros proyectos aquí expuestos cuentan con la visión de la comunidad y sobre esta visión formulan construyen y enriquecen los proyectos, todos tienen en cuanta la interdisciplinaridad de las áreas del conocimiento, pero no por su dialogo de saberes si no como una serie de actividades que debe cumplir cada una de ellas, en los tres primeros si se evidencia este dialogo entre las ciencias sociales (historia, economía, política), la cultura y las ciencias naturales.

Sin embargo, en el PRAE de la institución educativa villas del progreso dicha perspectiva interdisciplinar se toma como un conjunto de acciones propuestas a desarrollar por la comunidad, en este caso, los docentes de diferentes áreas colaboran con la ejecución de actividades pero no existe una apropiación de la propuesta que permita fortalecer los procesos que hasta ahora se han querido emprender, así mismo, la falta de diálogo entre el PRAE y la comunidad que vive en la zona impide un verdadero impacto social, limitando los alcances del proyecto.

En Consecuencia, los planteamientos actuales de la educación en la post modernidad, están requiriendo que los currículos se adapten a las situaciones socio - ambientales actuales de cada región, es por esto que se hace necesario que el desarrollo de los problemas ambientales tenga en cuenta dichas dimensiones, sin desligar una de la otra, debido a que las unas pueden ser 
consecuencia de las otras en una relación causa - efecto. (Sauve, 1999) Lo que no se evidencia en la construcción y desarrollo del PRAE evaluado. También es claro, que existe una corriente ambiental de tipo Conservacionista/recursista actual, pues este PRAE muestra su preocupación por preservar la calidad y la cantidad de los recursos disponibles en el colegio, así como la reutilización de residuos, mostrando al medio ambiente como una colección de recursos que se deben cuidar para el bien de los seres humanos (Sauve, 2005), olvidando a la naturaleza como otro del cual nos hemos beneficiado y al cual hemos desfavorecido (Leff, 2012).

Uno de los aspectos que no es abordado en la construcción de los PRAE es los diversos ambientes sociales en los cuales se desarrollan los estudiantes, ya que los seres humanos nos desarrollamos en ambientes físicos de los cuales tomamos provecho, pero también de ambientes sociales que hemos construido a lo largo de nuestra historia, estos ambientes sociales diversos determinan el comportamiento y forma de actuación frente a un grupo social determinado, por lo cual el estudio ambiental debe ir más allá del estudio físico y empezar a evaluar las diversas relaciones que se generan en todos los campos (Pérez, 2004).

Los proyectos que se destacaron al principio reconocen dentro de su formulación y diagnóstico del problema los factores físicos propios del ambiente en el cual se desarrollan, sin olvidar la parte estructural propia de la ciudad, y el concepto de calidad de vida, sin embargo, en el último, estos factores son descritos de manera muy superficial y reconocen dentro de la categoría de calidad solo los recursos presentes en el colegio sin evaluar su calidad, también se omite la forma en la cual está construida el colegio y que es una de los factores que afecta las relaciones que los estudiantes tienen con su ambiente, de estos requerimientos del espacio físico solo se reconoce la falta de espacios verdes, dificultad que si intento superar ubicando plantas en diferentes puntos de la institución, pero al no ser conscientes de la relación entre las plan tas y los estudiantes muchas de ellas terminaron dañadas, actualmente las pocas plantas que queda sirven como adorno pero en múltiples ocasiones se ven afectadas por el trato que reciben de los estudiantes (Pérez, 2004). 
Así cuando el espacio físico es limitado y quienes disponen de él no encuentran un sentido de pertenencia o identidad con el mismo, no se logra una relación armónica entre los elementos de la naturaleza y los elementos de la sociedad, y las relaciones sociales son el reflejo de las que se tienen con la naturaleza, pues al encontrarse en espacios hostiles por condiciones como:

Falta de espacios verdes, deportivos y de recreación

- Afinamiento en las aulas y patios de descanso

En ocasiones falta de salones

Las relaciones con el ambiente físico se tornan complejas y afectan el ambiente y las relaciones sociales, lo mismo ocurre en sentido contrario las relaciones sociales afectan las relaciones ambientales, en una comunidad como la del colegio en cuestión las situaciones familiares, personales y locales suelen afectar la manera en la cual los estudiantes se relacionan en el colegio ejemplo: un estudiante que es golpeado en casa, suele generar relaciones negativas frente a sus compañeros repitiendo el patrón o dejando que lo agradan, los mismo ocurre con su relación con el ambiente pues algunos de estos estudiantes suelen tener relaciones de poco o ningún respeto por su entorno.

\section{UNA PROPUESTA HACIA NUEVAS PRÁCTICAS EN AMBIENTALES ESCOLARES}

A pesar de que el panorama no es muy alentador en la formulación de los PRAE se puede decir que existen pocas experiencias exitosas, de las cuales podríamos tomar grandes ideas y empezar a generar propuestas más hacia el empoderamiento ambiental, el rescate de saberes ancestrales y populares; el uso del conocimiento científico, el reconocimiento de las realidades locales y las realidades personales de una población para construir una nueva percepción y relación con el ambiente escolar. En este sentido se plantea la siguiente propuesta para llegar a dicho proceso, sin embargo, como se indica anteriormente es solo una de las muchas que pueden surgir. (figura 2) 


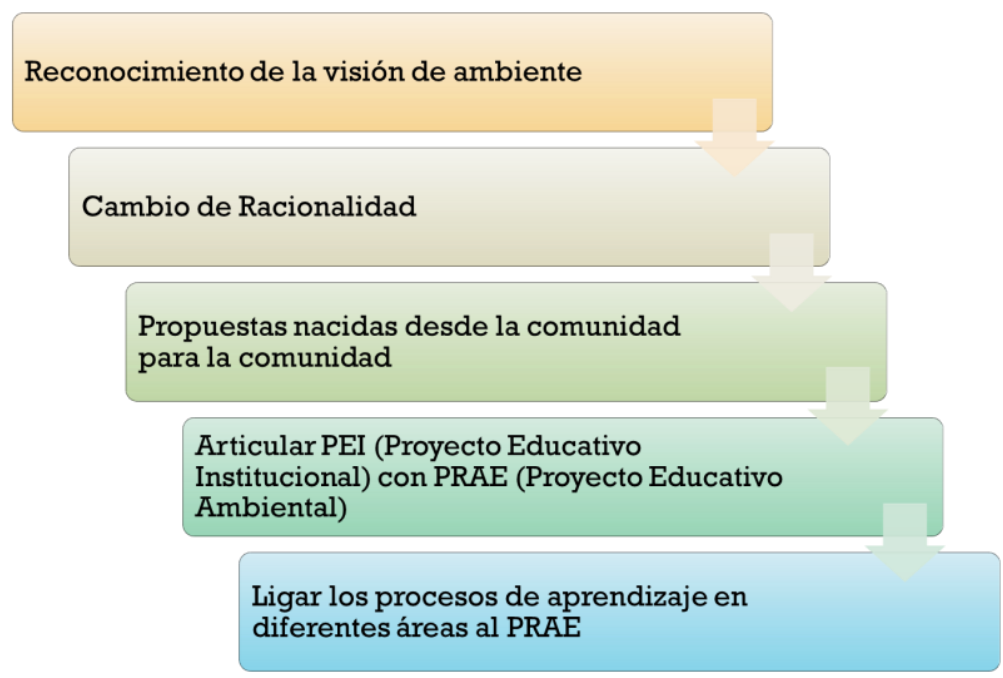

Figura 2. Sugerencia para lograr el cambio de racionalidad ambiental que permitan en empoderamiento ambiental. Elaboración propia

\section{Reconocimiento de la visión de Ambiente}

Antes de iniciar un proceso en educación ambiental es importante reconocer la concepción de ambiente y educación ambiental en los diferentes actores que participan del proceso, para este fin Sauve (2005), plantea la creación de un mapa del territorio pedagógico, en el cual los participantes del proceso reconozcan sus visiones del ambiente, y luego las agrupen en categorías según las características de cada una, reconociendo en ellas las divergencias, puntos comunes, oposición y complementariedad. Para con esto llegar a un diagnostico de cómo se están realizando las diversas practicas con respecto al ambiente en la institución educativa y en la comunidad en general.

Entendiendo que a partir de estas visiones se construye en colectivo una visión de ambiente, la cual debe ser fortalecida o ampliada para generar un proyecto ambiental que impacte de manera real a la comunidad, este servirá de punto de partida para general un cambio en la racionalidad ambiental y entender al ambiente como otro (Leff, 2012). 


\section{Cambio de la Racionalidad Ambiental}

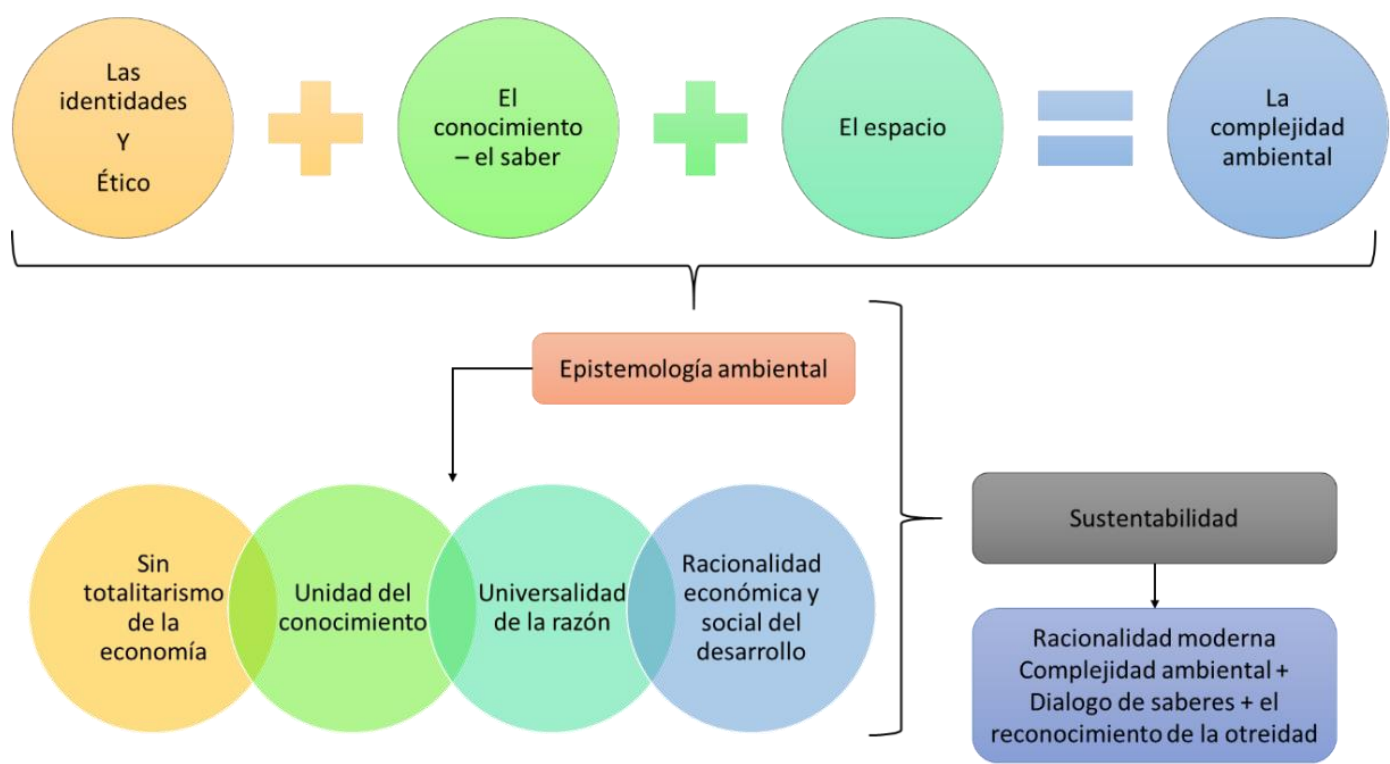

Figura 3. Pasos para el Cambio de Racionalidad Ambiental desde Enrique Leff. Tomado y adaptado de (Leff,2012)

Los proyectos ambientales deben cambiar la racionalidad frente al ambiente, es decir se debe:

Reconocer la identidad tanto individual como socio - cultural de las personas,

Debe existir un dialogo de saberes que permite la creación, desarrollo y funcionamiento de los proyectos ambientales, esto incluye directivos, docentes, estuantes, padres de familia, y sectores productivos de la zona,

Se deben entender las relaciones de armonía y tención presentes entre las personas y los diferentes ambientes (uno mismo, los otros el medio ambiente).

De esta forma se construirá una nueva epistemología ambiental, que permita a los sujetos entender al ambiente como otro con dinámicas propias, que se ven afectadas por la intervención del hombre, entendiendo con ello la complejidad de los procesos ambientales y su relación con lo político, económico, cultural y social. 
Este entendimiento del ambiente se puede hacer desde la perspectiva propia planteada por el colegio en la cual se puede fortalecer las dimensiones humanas y relacionarlas con el ambiente, así el Colegio Villas del Progreso trabaja por ciclos, en cada uno de los ciclos se trabaja de acuerdo con temáticas, ejes se pueden generar acciones que permitan el cambio de la racionalidad frente al ambiente así:

\begin{tabular}{|c|c|c|}
\hline $\begin{array}{l}\text { Ciclo / } \\
\text { Grados }\end{array}$ & Estructura General & Propuesta \\
\hline $\begin{array}{l}\text { Inicial/ } \\
\text { Jardín y } \\
\text { Transición }\end{array}$ & $\begin{array}{l}\text { Este ciclo tiene como fin la } \\
\text { construcción de sujetos y } \\
\text { desarrollo de la infancia a } \\
\text { partir del juego. } \\
\text { Dicho desarrollo de la } \\
\text { infancia tiene en cuenta las } \\
\text { diversas dimensiones (social, } \\
\text { ambiental, artística, } \\
\text { espiritual, etc.) }\end{array}$ & $\begin{array}{l}\text { Las actividades a realizar con los niños de ciclo } \\
\text { inicial deben propender desde el PRAE deben } \\
\text { propender por la realización de juegos de reglas } \\
\text { que permitan entender la lógica del ambiente, } \\
\text { ejemplo; si se juega a los animales los niños } \\
\text { deben entender la relación de cuidado y respeto } \\
\text { de esos otros, así como por dinámicas que } \\
\text { permitan entender la importancia de su ambiente } \\
\text { personal (como cuido de mí y del otro sin } \\
\text { importar su origen o especie) }\end{array}$ \\
\hline $\begin{array}{l}\text { Ciclo } 1 \text { / } \\
\text { 1ro y 2do }\end{array}$ & $\begin{array}{l}\text { EL JUEGO COMO } \\
\text { HERRAMIENTA } \\
\text { PEDAGÓGICA Y } \\
\text { DIDÁCTICA: Parte de } \\
\text { reconocer las características } \\
\text { personales, sociales y } \\
\text { contextuales de los niños y } \\
\text { niñas dando cuenta de su } \\
\text { desarrollo en los procesos de } \\
\text { pensamiento y las } \\
\text { tendencias de aprendizaje. } \\
\text { Fundamenta sus propósitos } \\
\text { alrededor del juego }\end{array}$ & $\begin{array}{l}\text { En este caso el mecanismo sigue siendo el } \\
\text { juego, por lo cual se deben establecer juegos de } \\
\text { reconocimiento de sí mismo como un ambiente } \\
\text { que se relaciona con otros. } \\
\text { Se pueden proponer juegos en los cuales los } \\
\text { estudiantes expongan actitudes de respeto por sí } \\
\text { mismo, el otro y su entorno. Así como actitudes } \\
\text { a auto cuidado mediante la comprensión del } \\
\text { ambiente, ejemplo: si el espacio es limitado y } \\
\text { existe riesgo de golpear a los demás y de dañar } \\
\text { las pocas zonas verdes se deben proponer } \\
\text { acciones pasivas que mitiguen los daños, } \\
\text { siempre guiadas hacia el respeto por la } \\
\text { diferencia. }\end{array}$ \\
\hline Ciclo 2 / & PROYECTO DE AULA: EI & Incentivar a los niños y niñas hacia la \\
\hline
\end{tabular}




\begin{tabular}{|c|c|}
\hline 3ro y 4to & $\begin{array}{l}\text { objetivo es que los niñas y } \\
\text { niñas se reconozcan como } \\
\text { protagonistas de su proceso } \\
\text { de aprendizaje, para ello se } \\
\text { han implementado cuatro } \\
\text { fases en la ruta } \\
\text { metodológica, abogando por } \\
\text { una educación diferenciada } \\
\text { en cuanto reconoce que los } \\
\text { infantes tienen diferentes } \\
\text { ritmos y formas de aprender. }\end{array}$ \\
\hline $\begin{array}{l}\text { Ciclo } 3 / \\
5 \text { to, 6to y } \\
7 \mathrm{mo}\end{array}$ & $\begin{array}{l}\text { ENFOQUE SOCIOCRÍTICO } \\
\text { PARTIENDO DE EJES } \\
\text { GENERADORES Y } \\
\text { APRENDIZAJE BASADO DE } \\
\text { PROYECTOS: Se plantean } \\
\text { unos postulados teóricos } \\
\text { desde el paradigma } \\
\text { sociocrítico cuyo enfoque se } \\
\text { centra en los niños, niñas y } \\
\text { adolescentes, propendiendo } \\
\text { por un cambio social desde } \\
\text { el interior de la comunidad } \\
\text { del ciclo. }\end{array}$ \\
\hline $\begin{array}{l}\text { Ciclo } 4 \text { / } \\
8 \text { vo y 9no }\end{array}$ & $\begin{array}{l}\text { PEDAGOGÍA DE LA } \\
\text { HUMANIZACIÓN: Evidencia } \\
\text { una mirada desde } \\
\text { Pedagogía de la } \\
\text { Humanización de Pablo } \\
\text { Romero Ibáñez, lo que } \\
\text { implica el reconocimiento de } \\
\text { las necesidades } \\
\text { cognitivas, comunicativas y } \\
\text { valores éticos de los } \\
\text { estudiantes del ciclo. }\end{array}$ \\
\hline $\begin{array}{l}\text { Ciclo } 5 \text { / } \\
10 \mathrm{mo} \text { y } 11\end{array}$ & $\begin{array}{l}\text { APRENDIZAJE BASADO EN } \\
\text { LA INVESTIGACION: La } \\
\text { enseñanza basada en la } \\
\text { investigación hace referencia } \\
\text { al diseño del programa } \\
\text { académico donde los } \\
\text { estudiantes requieren hacer } \\
\text { conexiones intelectuales y } \\
\text { practicas entre el contenido y } \\
\text { las habilidades. }\end{array}$ \\
\hline
\end{tabular}

formulación de proyectos respecto al ambiente, relaciones de los niños consigo mismo, con otros y sus entornos físicos con temas cercanos y que puedan despertar la curiosidad de los estudiantes, algunos ejemplos pueden ser: Origami como un arte ancestral y su contribución con el ambiente, higiene personal y su importancia, porque es importante la separación de los residuos, etc.

Esta curiosidad se puede despertar desde las realidades cercanas de los niños con cosas como lecturas, cuentos, experiencias y experimentos, así como las mismas historias de padres y profesores

Este ciclo presenta una particularidad y son los temas que rigen cada uno de los grados y son transversales a todas la asignaturas Colombia, América y el Mundo, en este sentido se pueden generar nuevas racionalidades en los estudiantes si se plantea u conocimiento de la historia y los fenómenos que han llevado a la crisis ambiental actual y a partir de estas problemáticas evaluar cuales son las consecuencias ambientales presentes en la zona, alguna de las propuestas de los niños, niñas y adolescentes pueden servir situación central sobre la cual se desarrolle el PRAE Prácticas en las cuales se establezcan relaciones más armónicas entre los estudiantes y el ambiente, en donde la humanización implique el entendimiento de otros lenguajes y permite el dialogo de saberes en la construcción de contenidos sobre el espacio físico y los ambientes, que reconozcan cuáles son sus realidades y como estas afectan sus relaciones sociales y ambientales dentro de la institución y fuera de ella. Ejemplo: Reconocer la emocionalidad, simbología y el territorio en sus diversas dimensiones a partir de la cartografía social.

Incentivar en los estudiantes de grado decimo y once la elaboración de proyectos de investigación desde la parte ambiental nacidos del reconocimiento de las relaciones que ha generado la comunidad con el ambiente. Que sean parte activa y proponente de nuevas formas de dinamizar el PRAE dentro y fuera de la comunidad generando impacto.

Cuadro 2. Actividades propuestas para el desarrollo del PRAE en la Institución Educativa Distrital Colegio Villas del Progreso 


\section{Elección de las Problemáticas Ambientales}

El cambio de racionalidad sobre el ambiente llevara poco a poco a los estudiantes a reconocer las situaciones ambientales que reconocen, es a partir de este reconocimiento y de las necesidades propias de los estudiantes que deben elegirse las problemáticas que permitan dinamizar el PRAE, en este punto se deben generar canales de participación de la mano con el comité ambiental y el gobierno escolar que permitan formular las soluciones y canalizar todas las propuestas, es importante en una comunidad tan rica culturalmente incentivar el dialogo de saberes para el desarrollo del proyecto.

\section{CONCLUSIONES}

Los PRAE constituyen desde la normatividad un instrumentos sumamente completos y articulados que permiten la educación ambiental en la formalidad, sin embargo, muchos se quedan cortos o no generan un alto impacto en las comunidades en las cuales se desarrollan, en este sentido, el PRAE debe propender por un cambio en la racionalidad ambiental de las comunidades para que de esta forma se logre el impacto esperado.

Otra de sus funciones debe ser la de educar a la comunidad hacia una nueva visión del ambiente que tenga en cuenta las relaciones consigo mismo, el otro y el entorno, la propuesta expuesta aquí es solo el inicio de un arduo trabajo que se debe llevar a cabo por la institución educativa que se está evaluando, para que a partir de este primer paso sea la misma comunidad educativa en su conjunto quienes se empoderen de sus realidades y busquen soluciones acorde con las situaciones sociales y culturales que allí se presentan. 


\section{REFERENCIAS}

Colombia. Política Nacional de Educación Nacional PNEN: SINA. (2002). Bogotá.

García, J y Ferrandis I (1990). Revisión Histórica del Concepto de Educación Ambiental. Revista Didáctica de la Ciencias Experimentales y Sociales, $\mathrm{N}^{\circ} 3$

Leff. E. (2012) Pensamiento ambiental latinoamericano: patrimonio de un saber para la sustentabilidad. Environmental Ethics, v.34, n. Suplemento. p. 97-112

Novo, M. (2006). El desarrollo sostenible. Su dimensión ambiental y educativa. Pearson Educación S.A

Sauvé, L. (2005). Uma cartografia das corrientes em educação ambiental. (p. 1746). In Sato, M.et Carvalho, I. (Dir.). Educação ambiental - Pesquisa e desafíos. Porto Alegre: Artmed.

Sauvé, L. (2009). “Un mensaje de Lucie Sauvé, presidente" en La Tierra, hogar de todos. $5^{\circ}$ Congreso Mundial de Educación Ambiental. Montreal, mayo 10-14. En línea: http://www.5weec.uqam.ca/ES/mot_copresidents.asp 
Sauvé, L. (2012): "Cruzar las dimensiones críticas, ética y política de la educación ambiental: hacia una ecociudadanía" en Cuarto Encuentro Nacional de Educación Ambiental para el Desarrollo Humano Sustentable, Montevideo. En línea:

http://esdocs.com/doc/126511/revista---ministerio-de-educaci\%C3\%B3n-y-cultura--Uruguay. 